\title{
Dynamics of the mean transmitral pressure gradient and its impact on clinical outcomes after MitraClip
}

\author{
Can Öztürk ${ }^{1}$, Kim Sprenger ${ }^{1}$, Noriaki Tabata ${ }^{1}$, Atsushi Sugiura ${ }^{1}$, Marcel Weber ${ }^{1}$, Georg \\ Nickenig ${ }^{1}$, and Robert Schueler ${ }^{2}$ \\ ${ }^{1}$ Universität Bonn \\ ${ }^{2}$ Elisabeth-Krankenhaus-Essen GmbH Klinik für Kardiologie und Angiologie
}

February 2, 2021

\begin{abstract}
Background: The impact of the increased mitral gradient (MG) on outcomes is ambiguous. Therefore, we aimed to evaluate a) periinterventional dynamics of MG, b) the impact of intraprocedural MG on clinical outcomes, and c) predictors for unfavourable MG values after MitraClip. Methods: We prospectively included patients undergoing MitraClip. All patients underwent echocardiography at baseline, intraprocedurally, at discharge, and after six months. 12-month survival was reassessed. Results: 175 patients (age $81.2 \pm 8.2$ years, $61.2 \%$ male) with severe mitral regurgitation (MR) were included. We divided our cohort into two groups with a threshold of intraprocedural $\mathrm{MG}$ of $4.5 \mathrm{mmHg}$, which was determined by the multivariate analysis for the prediction of 12-month mortality ( $<4.5 \mathrm{mmHg}$ : Group 1, ?4.5 mmHg: Group 2). Intraprocedural $\mathrm{MG} ? 4.5 \mathrm{mmHg}$ was found to be the strongest independent predictor for 12-month mortality (HR: 2.33, p=0.03, OR: 1.70, p=0.05) and [?]3.9 $\mathrm{mmHg}$ was associated with adverse functional outcomes (OR: 1.96, $\mathrm{p}=0.04)$. The baseline leaflet-to-annulus index ( $>1.1$ ) was found to be the strongest independent predictor (OR: 9.74, $\mathrm{p}=0.001$ ) for unfavourable intraprocedural $\mathrm{MG}$, followed by the number of implanted clips $(\mathrm{p}=0.01), \mathrm{MG}$ at baseline $(\mathrm{p}=0.02)$ and central clip implantation $(\mathrm{p}=0.05)$. Conclusion: MG shows time-varying and condition-depended dynamics periinterventionally. Patients with persistent increased ([?]4.5 mmHg) MG at discharge showed the worst functional outcomes and the highest 12-month mortality, followed by patients with an intra-hospital decrease in MG to values below $4.5 \mathrm{mmHg}$. Pre-interventional echocardiographic and procedural parameters can predict unfavourable postprocedural MG.
\end{abstract}

Dynamics of the mean transmitral pressure gradient and its impact on clinical outcomes after MitraClip

Can Öztürk ${ }^{1} M D$, Kim Sprenger ${ }^{1} M D$, Noriaki Tabata ${ }^{1}$ MD, Atsushi Sugiura ${ }^{1}$ MD, Marcel Weber ${ }^{1}$ MD, Georg Nickenig ${ }^{1} M D$, Robert Schueler ${ }^{2} M D$

${ }^{1}$ Department of Cardiology, University Hospital Bonn, Bonn, Germany

${ }^{2}$ Contilia Heart and Vascular Center, Elisabeth Hospital, Essen, Germany

Running head: Mitral gradient predicts outcomes after MitraClip

Address for correspondence:

Can Öztürk, $M D$

Heart Center, Department of Cardiology

University Hospital Bonn 
Venusberg-Campus 1, Building 26

53127 Bonn

Germany

Tel +49-228-287-16670

Fax $+49-228-287-19010$

ORCID: 0000-0002-5419-2488

\section{ABSTRACT}

\section{Background:}

The impact of the increased mitral gradient (MG) on outcomes is ambiguous. Therefore, we aimed to evaluate a) periinterventional dynamics of MG, b) the impact of intraprocedural MG on clinical outcomes, and c) predictors for unfavourable MG values after MitraClip.

\section{Methods:}

We prospectively included patients undergoing MitraClip. All patients underwent echocardiography at baseline, intraprocedurally, at discharge, and after six months. 12-month survival was reassessed.

\section{Results:}

175 patients (age $81.2 \pm 8.2$ years, $61.2 \%$ male) with severe mitral regurgitation (MR) were included. We divided our cohort into two groups with a threshold of intraprocedural $\mathrm{MG}$ of $4.5 \mathrm{mmHg}$, which was determined by the multivariate analysis for the prediction of 12-month mortality (<4.5 mmHg: Group 1, [?] $4.5 \mathrm{mmHg}$ : Group 2).

Intraprocedural MG [?] $4.5 \mathrm{mmHg}$ was found to be the strongest independent predictor for 12-month mortality (HR: $2.33, \mathrm{p}=0.03$, OR: $1.70, \mathrm{p}=0.05$ ) and [?] $3.9 \mathrm{mmHg}$ was associated with adverse functional outcomes (OR: $1.96, \mathrm{p}=0.04)$.

The baseline leaflet-to-annulus index (>1.1) was found to be the strongest independent predictor (OR: 9.74, $\mathrm{p}=0.001)$ for unfavourable intraprocedural $\mathrm{MG}$, followed by the number of implanted clips $(\mathrm{p}=0.01)$, MG at baseline $(\mathrm{p}=0.02)$ and central clip implantation $(\mathrm{p}=0.05)$.

\section{Conclusion:}

MG shows time-varying and condition-depended dynamics periinterventionally. Patients with persistent increased ([?]4.5 mmHg) MG at discharge showed the worst functional outcomes and the highest 12-month mortality, followed by patients with an intra-hospital decrease in $\mathrm{MG}$ to values below $4.5 \mathrm{mmHg}$. Preinterventional echocardiographic and procedural parameters can predict unfavourable postprocedural MG.

KEYWORDS : MitraClip, mean pressure gradient, mitral regurgitation, outcome

\section{INTRODUCTION}

Transcatheter edge-to-edge mitral valve (MV) repair with the MitraClip system is increasingly regarded as a successful and effective therapeutic alternative to surgical therapy for relevant refractory mitral regurgitation (MR) in patients at high surgical risk (1), (2), (3). The MitraClip procedure reduces the MV area and generates, at least two new orifices, followed by an increase of the mean transmitral pressure gradient (MG). An MG over five mmHg after clip attachment has been shown to be associated with adverse outcomes and should thus be avoided according to the current guidelines (4), (5). On the contrary, some recent studies found no predictive value of MG for clinical outcomes after interventional therapy for functional MR (6), (7). 
MG is assessed by transesophageal echocardiography using the MV peak-systolic velocity from intraprocedural continuous-wave Doppler measurements. Intraprocedural assessment of MG can be influenced by various factors: heart rate and rhythm, hemodynamics during general anaesthesia and presence of inotropes, as well as measurement-related factors, such as angulation errors. Furthermore, there are additional heart-related factors: (i) left-atrial compliance, (ii) left-ventricular end-diastolic pressure, and (iii) valvular parameters (8), (9), (10). Therefore, intraprocedural MG should be carefully and individually anticipated, since the cofactors mentioned are dynamic and time-varying parameters and might lead to over-or underestimation of MG. Taken together, it is unknown how much the intraprocedurally measured MG values change following general anaesthesia and restoration of "normal" hemodynamic conditions or following epithelialisation of the clip devices.

We, therefore, aimed to a) evaluate the dynamic changes of MG, both peri-interventionally and during the follow-up (FU), b) assess the impact of periinterventionally measured MG on clinical outcomes, and c) analyse predictors for unfavourable MG after MitraClip.

\section{METHODS:}

\section{Patients, follow-up, and endpoints:}

We prospectively included consecutive patients with symptomatic moderate-to-severe or severe refractory MR undergoing the MitraClip? procedure (NTR/XTR Clip Delivery System, Abbot Vascular, Inc., Santa Clara, California) at the Heart Center of the University Hospital Bonn between February 2017 and January 2019 .

All patients underwent standardised echocardiographic examinations for non-invasive MG assessments at baseline, intraprocedurally, at discharge, and six months after MitraClip. Multiple measurements were done to minimise angulation- and acquisition-related errors and exclude any relevant mitral valve stenosis $(>5$ $\mathrm{mmHg}$ ) during each examination, or directly after clip deployment and before clip release. All echocardiograms were performed after a relaxing time of 5 minutes to occasion a resting condition to avoid misinterpretations due to hemodynamic undulations. Clinical examinations comprised an assessment of NYHA functional class, a six-minute walk test (6MWT), and a comprehensive blood test, which included serum levels of NT-proBNP. The six-month FU was performed in our outpatient clinic and included transthoracic echocardiography, a routine physical examination, an electrocardiogram, and a blood test. Survival status was reassessed by either a FU visit in the outpatient clinic or a phone call 12 months after the procedure.

We defined all-cause mortality at 12-month FU as the primary endpoint in line with MVARC (Mitral Valve Academic Research Consortium) definitions (11). Secondary endpoints were defined as follows: NYHA functional class at FU < III, amelioration in the walk distance of $25 \%$, intraprocedural MG [?] $4.5 \mathrm{mmHg}$, residual $\mathrm{MR}>\mathrm{II}$ at discharge, and $\mathrm{MR}$ at $\mathrm{FU}>\mathrm{II}$.

The study was authorised by the local ethics committee (Medical Faculty of University Bonn, Bonn, Germany) and in accordance with the Declaration of Helsinki. All patients signed their written informed consent before inclusion in the study. All patients' data were anonymised before use in the study. Echocardiographers and clinicians from the in- and outpatient clinics were blinded to the study parameters. Trained study nurses carried out clinical FU evaluation, unattended by the interventionalists or the procedural echocardiographer.

\section{Defining the groups:}

According to multivariate regression analysis and a receiver operating characteristic (ROC) analysis, we found intraprocedural $\mathrm{MG}$, with a cut-off value of $4.5 \mathrm{mmHg}$, was the strongest predictor of the primary endpoint (Table 5) . Consequently, we divided our patient cohort into two groups - patients with an intraprocedural $\mathrm{MG}<4.5 \mathrm{mmHg}$ were defined as group 1 and [?] $4.5 \mathrm{mmHg}$ asgroup 2 .

Additionally, we divided the patients from group 2 into two further groups: group $2 A$ represents patients with persistent MG [?]4.5 $\mathrm{mmHg}$ at discharge. Group $2 B$ consists of patients with an in-hospital decrease of MG below $4.5 \mathrm{mmHg}$. 


\section{Echocardiographic assessment:}

Echocardiographic assessments were performed in line with the current recommendations and guidelines of the European Association of Echocardiography, including comprehensive echocardiography (12). The severity of MR was assessed by the semi-quantitative PISA-method, using the radius of proximal isovelocity surface area (PISA radius), the effective regurgitant orifice area (EROA), as well as the vena contracta width (VC) and the regurgitant volume (RegVol) (13). MG was estimated from the peak-systolic velocity from continuous-wave Doppler imaging of the MV. Right ventricular systolic pressure (RVSP) was estimated by the tricuspid systolic-peak velocity using the modified Bernoulli equation (instantaneous pressure gradient $(\Delta \mathrm{P})=4 \mathrm{x}$ velocity). The leaflet-to-annulus index (LAI) was calculated by a formula defined as the ratio between the summation of the lengths of the mitral valve leaflets (anterior mitral leaflet + posterior mitral leaflet) and the anteroposterior diameter (AP-diameter), as published previously (14). The leaflet lengths and AP-diameter were measured at $120-150^{\circ}$ (three-chamber view) and SL-diameter at $0-20^{\circ}$ (four-chamber view) in transesophageal echocardiography. The echocardiographic studies were performed with currently available ultrasound machines (iE33, Philips Medical Systems, Andover, Massachusetts; E9, GE Healthcare Vingmed Ultrasound, Horten, Norway).

\section{Statistical analysis}

The normal distribution of continuous variables was examined using the Shapiro-Wilk test. Continuous data were expressed as mean values \pm the standard deviation if normally distributed. Categorical data were presented as a percentage. The Student's two-sample t-test or the Man-Whitney U test was performed to compare continuous variables. Fisher's exact test or the Chi-square test was used to compare categorical data. To compare more than two variables, we used the one-way variance analysis or the Kruskal-Wallis test as an extension of the Student's t-test. Univariate analysis was performed to assess the predictors of clinical outcomes. The predictors of 12-month mortality were estimated by multivariate regression analysis. Cumulative survival incidence was compared using the Log-rank test between the groups and presented by the Kaplan-Meier curve. A ROC analysis was performed to determine independent predictors' sensitivity and specificity for unfavourable outcomes and mortality with defined cut-off values. Two-tailed p-values were considered to be significant if ranging below 0.05. Statistics were performed using SPSS (PASW statistic, Version 25.0.0.0, SPSS Inc., Chicago, Illinois, USA) and MedCalc Statistical Software (Version 19.2, MedCalc Software Ltd, Ostend, Belgium).

\section{RESULTS:}

\section{Baseline characteristics:}

175 consecutive patients (61.2\% male) with symptomatic (100\% NYHA functional class $>$ II), moderateto-severe or severe MR (PISA: $0.8 \pm 0.2 \mathrm{~cm}$, VC: $0.8 \pm 1.2 \mathrm{~cm}$, EROA: $0.5 \pm 0.3 \mathrm{~cm}^{2}$, RegVol: $51.1 \pm 19.7 \mathrm{ml}$ ) were included. $40 \%(n=70)$ of the patients showed degenerative MR (DMR), $42.8 \%(n=75)$ of patients had functional MR (FMR), and 17.2\% ( $\mathrm{n}=30)$ of patients had a mixed etiology. At baseline, all patients were on guideline-directed medical heart-failure therapy or device therapy, if needed. All patients were classified as inoperable or at a high surgical risk by the heart team owing to advanced comorbidities (Logistic EuroScore: $17.8 \pm 5.2 \%$ ), advanced age (mean age: $81.2 \pm 8.2$ years) and frailty assessed by clinicians' estimations.

Concerning the baseline demographical and clinical characteristics, there were no statistically significant differences between the groups. Of note, the serum level of NT-proBNP was more elevated in group 2 than group 1, but without reaching the level of statistical significance $(4121.9 \pm 3955 \mathrm{pg} / \mathrm{ml}$ vs $6121.5 \pm 8864.7$ $\mathrm{pg} / \mathrm{ml}, \mathrm{p}=0.1$ ). The baseline demographical and clinical characteristics are presented inTable 1 .

In the overall cohort, baseline echocardiography showed a relevant left-ventricular (LV) dilation (end-diastolic volume [LV-EDV]: $164.7 \pm 68.3 \mathrm{ml}$, end-systolic volume [LV-ESV]: $96.2 \pm 58.8 \mathrm{ml}$ ) with a decreased LV ejection fraction (LV-EF: $44.7 \pm 16.3 \%$ ). There was no relevant mitral valve stenosis observed at baseline $(1.5 \pm 1.1$ $\mathrm{mmHg})$. Furthermore, we found increased RVSP $(45.4 \pm 14.8 \mathrm{mmHg})$ as a sign of pulmonary hypertension at baseline. The baseline echocardiographic parameters were comparable between the groups (Table 2). 


\section{Interventional outcomes and technical success:}

All patients underwent a successful transcatheter edge-to-edge mitral valve repair using the MitraClip? NTR/XTR Delivery System with a reduction in MR of at least one grade or to an MR grade $<$ moderateto-severe as previously described (15), (16), (17). To maintain optimal procedural conditions and outcomes, all procedures were performed under general anaesthesia with controlled haemodynamic conditions such as systolic blood pressure in the range of $110-130 \mathrm{mmHg}$ and the heart rate between $60-80 \mathrm{bpm}$.

$54 \%(n=95)$ of patients received one clip device, $43 \%(n=75)$ of patients were treated using two clip devices. Three clip devices were implanted in $3 \%(n=5)$ of patients, without a significant difference in the arithmetic mean of the number of clip devices used between the groups; however, the one-clip approach was performed significantly more often in group $1(1.43+-0.58$ vs $1.41+-0.7, \mathrm{p}=0.9)$. There was no significant difference in post-procedural residual MR between the groups (MR < III: Group 1: 94.2\% (n=145) vs Group 2: 86\% $(\mathrm{n}=18), \mathrm{p}=0.3)$. No major periprocedural complications, such as pericardial tamponade or vascular injury requiring additional surgical or interventional therapy, occurred.

\section{Follow-up in all patients:}

Two patients (1.1\%) were deceased during the 30-day FU, due to cardiovascular causes. The six-month all-cause mortality rate was $8.5 \%(\mathrm{n}=15)$ and the 12 -month all-cause mortality rate was $23.4 \%(\mathrm{n}=41)$.

At six-month FU, we found significantly improved NHYA functional class (NYHA>II: 100\% to 31.66\%, $\mathrm{p}=0.001)$ and increased six-minute walk distance $(247.23+-33.4 \mathrm{~m}$ to $333.45+-60.5 \mathrm{~m}, \mathrm{p}=0.001)$ in the overall cohort.

Echocardiography at six-month FU showed no significant changes in LV dimensions and function (LV-EDV: group $1, \mathrm{p}=0.6$ vs group $2, \mathrm{p}=0.3$; LV-ESV: group $1, \mathrm{p}=0.4$ vs group $2, \mathrm{p}=0.5 ; \mathrm{LV}$-EF: group 1 , $\mathrm{p}=0.5$ vs group 2, $\mathrm{p}=0.9)$. We found a significant and sustained reduction in MR for the overall cohort six months after the procedure (MR $>$ II; $91.36 \%$ to $2.48 \%, \mathrm{p}<0.001)$. Moreover, RVSP significantly decreased within the six-month FU period $(45.4+-14.8 \mathrm{mmHg}, 34.7+-10.5 \mathrm{mmHg}, \mathrm{p}=0.01)$.

\section{Dynamic changes in MPG:}

MG significantly increased after the MitraClip procedure $(1.5+-1.1 \mathrm{mmHg}$ to $3.5+-1.7 \mathrm{mmHg}, \mathrm{p}=0.03)$ in the overall cohort. Elevated $\mathrm{MG}$ values above $4.5 \mathrm{mmHg}$ were measured intraprocedurally in 21 patients $(12 \%)$-group 2 .

Concerning MG's peri-interventional dynamics, we found a decrease in MG to values below $4.5 \mathrm{mmHg}$ in 11 patients (52\%) from group 2 at discharge - group $2 B$. In total, $94 \%$ of patients $(\mathrm{n}=165)$ presented with an $\mathrm{MG}<4.5 \mathrm{mmHg}$ and only $6 \%$ of patients $(\mathrm{n}=10)$ had persistent increased MG [?]4.5 $\mathrm{mmHg}$ at discharge group $2 A$.

At six-months FU, the following MG values were documented: (i) increase to values above $4.5 \mathrm{mmHg}$ in nine patients (5.45\%) from group 1 and (ii) decrease to values below $4.5 \mathrm{mmHg}$ in two patients (20\%) from group 2A, in addition to 11 patients with a pre-discharge reduction in MG. Overall, we found 146 patients (91\%) with $\mathrm{MG}<4.5 \mathrm{mmHg}$ and 14 patients (9\%) with MG [?]4.5 $\mathrm{mmHg}$ at six-month FU. The dynamics of MG values over time are presented in Figure 1.

\section{Comparison of the groups:}

Concerning baseline echocardiography, we found higher MG in group $2(1.5+-1 \mathrm{mmHg}$ vs $2.6+-1.1 \mathrm{mmHg}$, $\mathrm{p}=0.001)$, as expected. However, the MV geometry war comparable the groups - baseline AP-diameter (37.3+- $0.5 \mathrm{~mm}$ vs $37.5+-0.5 \mathrm{~mm}, \mathrm{p}=0.9)$ and baseline SL-diameter $(39.9+-6.9 \mathrm{~mm}$ vs $39.2+-5.9 \mathrm{~mm}$, $\mathrm{p}=0.9$ ). The remaining baseline echocardiographic parameters were statistically comparable between the groups. Of note, there were no significant differences concerning the aetiology of MR between the groups (Table 2). 
At six-month FU in survived patients $(\mathrm{n}=160)$, the NYHA functional class was found to be significantly improved in group 1 (NYHA >II; $100 \%$ to $26 \%$, p <0.001), but not in group 2 (NYHA >II; $100 \%$ to $73 \%$, $\mathrm{p}=0.5)$. We, furthermore, found an increased walk distance (6MWT) in group $1(252.2+-127.8 \mathrm{~m}$ to $348.3+-$ $80.7 \mathrm{~m}, \mathrm{p}=0.05)$, but this value was unchanged in group $2(210.8+-46.5 \mathrm{~m}$ to $223.3+-25.8 \mathrm{~m}, \mathrm{p}=0.3)$. Serum levels of NT-proBNP tended to decrease in group 1 and increase in group 2 at six-month FU (5043.4+-4039.1 $\mathrm{pg} / \mathrm{dl}$ to $4145.9+-3352.1 \mathrm{pg} / \mathrm{dl}$, p=0.3 vs. $5389.5+-1068.4 \mathrm{pg} / \mathrm{dl}$ to $9121.5+-8864.8 \mathrm{pg} / \mathrm{dl}, \mathrm{p}=0.6$ ) (Table 3).

We found a significant and sustained reduction in MR severity in both groups six months after the procedure (MR [?]III; $91 \%$ to $2 \%, \mathrm{p}<0.001,94 \%$ to $6 \%$, p $<0.001$ ). RVSP significantly decreased only in group 1 (44.6+$11.3 \mathrm{mmHg}$ to $33.3+-4.2 \mathrm{mmHg}, \mathrm{p}=0.016$ ). Patients with an intraprocedural $\mathrm{MG}$ [?]4.5 mmHg showed no significant RVSP changes $(49.4+-18.3 \mathrm{mmHg}$ to $45.6+-18.7 \mathrm{mmHg}, \mathrm{p}=0.5)$ at FU(Table 3) .

\section{Predictors for clinical outcomes:}

There was a statistically significant difference in 12-month mortality between the groups $(20.7 \%$ ( $\mathrm{n}=32)$ vs $42.8 \%(\mathrm{n}=9), \mathrm{p}=0.02)$. Survival status within the 12-month FU period is graphically depicted by the Kaplan-Meier curve (Figure 2). A Cox-regression analysis showed significantly higher 12-month mortality in group 2 compared to group 1 (HR: 2.33, 95\% CI: 1.11 to $4.88, \mathrm{p}=0.03$ ) and a multivariate analysis relieved that intraprocedural MPG is the strongest predictor (OR: 1.70, 95\% CI: 0.95 to 3.05, $\mathrm{p}=0.05$ ) for 12-month mortality compared to MGs at another time points, residual $\mathrm{MR}>\mathrm{II}$ at discharge and recurrent $\mathrm{MR}>\mathrm{II}$ at FU (Table 4) .

According to multivariate regression analysis, including ROC curve analysis of MGs at three different time points (intraprocedurally, at discharge, and six-month FU) concerning the prediction of adverse functional outcomes (NYHA at FU >II, improvement in walk distance $<25 \%$ ), we found intraprocedural MG to be the strongest predictor for unfavourable clinical outcomes (OR: $1.96,95 \%$ CI: 1.02 to $3.75, \mathrm{p}=0.04$ ) with a cut-off value of $3.9 \mathrm{mmHg}$ (specificity of $80 \%$ and sensitivity of $63.9 \%$ ) followed by MG at FU (OR: 1.56 , $95 \%$ CI: 0.85 to $2.86, \mathrm{p}=0.14$ ) with a cut-off value of $2.6 \mathrm{mmHg}$; however, this finding was without statistical significance(Supplementary Table 1).

According to the one-way variance analysis of group 1, group $2 \mathrm{~A}$, and group $2 \mathrm{~B}$ concerning adverse functional outcome - higher NYHA functional class ( $>$ II) and lower walk distance (improvement $<25 \%$ ) - we found a significant difference between the groups ( $24 \%$ vs $75 \%$ vs $55 \%$, p<0.001) (Figure 3A) . Group $2 \mathrm{~A}$ and 2B showed a somewhat higher 12-month mortality rate than group 1, but without statistical significance ( $40 \%$ vs 33\%, vs $29 \%$, p=0.4) (Figure 3B). Remarkably, the worst outcomes - not only functional but also mortality- occurred in group $2 \mathrm{~A}$.

\section{Predictors for unfavourable intraprocedural MPG:}

We performed a variance analysis using the Kruskal-Wallis test in patients who underwent one-clip implantation to evaluate the impact of clip orientation on intraprocedural MG in patients, who become only one-clip implantation. Central clip implantation (segment A2-P2) was found to induce higher intraprocedural MG $(3.58+-1.7 \mathrm{mmHg}$ ), followed by the technique with cross-clipping (i.e., segment A2-P3, A2-P1; 3.2+-1.7 $\mathrm{mmHg}$ ). Non-central non-cross clip implantation (segment A3-P3; 2.98+-1.3 mmHg or A1-P1; 2.66+-1.04 $m m H g$ ) led to a lower MG after clipping in our cohort (Supplementary Figure 1) .

Furthermore, we performed an analysis of variance (ANOVA) test to assess the impact of MR aetiology on intraprocedural MG in patients with the one-clip approach. It showed that patients with degenerative or mixed MR had higher intraprocedural MG (Supplementary Figure 2) .

To further evaluate our data for predictors of unfavourable intraprocedural MG, we analysed the anatomical features of the MV. According to linear regression analysis, the AP-diameter had a significant negative correlation with intraprocedural MG in patients who underwent one-clip implantation ( $\mathrm{r}=3.75,95 \%$ CI: 3.57 to $3.94, \mathrm{p}<0.001$ ). Thus, patients with smaller annular dimensions (cut off value: $38 \mathrm{~mm}$ ) had higher intraprocedural MG values. In addition, the leaflet-to-annulus index (LAI) $(<1.11)$ was found to be a strong 
predictor (AUC: $0.595,95 \%$ CI: 0.542 to $0.647, \mathrm{p}=0.0031$ ) for unfavorable intraprocedural $\mathrm{MG}(>4.5 \mathrm{mmHg}$ ) and showed a significant negative correlation with intraprocedural MG values in the regression analysis (OR: $18.4395 \%$ CI: 8.86 to $125.49, \mathrm{p}=0.0029)$. According to the multivariate regression analysis, we found LAI (OR: 9.74, 95\% CI: 0.43 to $217.17, \mathrm{p}=0.001$ ) to be the strongest, independent predictor for unfavorable intraprocedural MG ([?]4.5 mmHg) followed by the count of implanted clips > 2 (OR: 7.54, 95\% CI: 1.24 to $87.67, \mathrm{p}=0.01$ ), MG at baseline (OR: $2.06,95 \% \mathrm{CI}: 1.58$ to $2.68, \mathrm{p}=0.02$ ) and central clip implantation (OR: $1.87,95 \%$ CI: 0.67 to $3.45, \mathrm{p}=0.05$ ) (Figure 4 ) .

\section{DISCUSSION:}

The major findings of the present study are as follows:

1. Intraprocedural MG was found to be a strong predictor for 12-month mortality (cut-off value: 4.5 $\mathrm{mmHg}$ ) and adverse functional outcomes (cut-off value: $3.9 \mathrm{mmHg}$ ).

2. We found the worst functional outcomes assessed by NYHA functional class and walk distance as well as the highest 12-months mortality in patients with persistent MG [?] $4.5 \mathrm{mmHg}$ at discharge independently from the MR aetiology followed by in patients with an in-hospital decrease in MG to values below $4.5 \mathrm{mmHg}$ at discharge.

3. LAI $(<1.11)$ was found to be the strongest predictor for unfavourable intraprocedural MG $(>4.5$ $\mathrm{mmHg}$ ) followed by baseline $\mathrm{MG}$, the number of implanted clips, and central clip localisation.

Elevated MG following the MitraClip procedure is considered to be associated with adverse outcomes (20). Therefore, the current guidelines recommend avoiding an intraprocedural transmitral pressure gradient above five $\mathrm{mmHg}$ (11). On the other hand, some contradicting studies currently show no relevant association between MG and outcomes after the MitraClip procedure, particularly in patients with functional MR (6), (7). Therefore, the effect of MG on outcomes after MitraClip is ambiguous.

Intraprocedural assessment of mitral inflow patterns and hemodynamics might be challenging owing to altered mitral geometry, such as the double- or multi-orifice MVs after transcatheter MV repair. Additionally, intraprocedurally assessed MG might be altered by "abnormal" hemodynamic conditions through general anaesthesia or inotropes. Despite difficulties in MG assessment by using continuous-wave Doppler in the setting of catheter-based MV repair, it was proven to be superior over planimetric evaluation of MVA for stenosis assessment, in a study with 38 patients by Biaggi and coworkers. They found peri-interventional planimetry to be unacceptably time-consuming and also to underestimate the MVA due to technical and acquisition-related issues (18). Accordingly, we used the Doppler-based assessment of MG in the present study.

A linear relationship between intraprocedural $\mathrm{MG}>5 \mathrm{mmHg}$ and decreased functional capacity, as assessed by using the NYHA functional classification, was demonstrated by Thaden et al. in a cohort with $90 \%$ DMR $(\mathrm{n}=112)$ and preserved LV function (mean LV-EF: 50\%). Correlatively, MG at baseline, the number of implanted clips, and central clip implantation were shown to be independent predictors for high intraprocedural MG in our study (21).

Neuss et al. found an invasively measured $\mathrm{MG}>5 \mathrm{mmHg}$ and an echocardiographically estimated MG $>4.4 \mathrm{mmHg}$ to be independent predictors for all-cause mortality in 268 patients (mean age: 75 years) with moderately reduced LV-function (mean LV-EF: 39\%) and moderate-to-severe or severe MR, who underwent the MitraClip procedure (19). Despite some differences in clinical and echocardiographic characteristics, such as older patients with better LV-function and a lower FMR rate, their findings support the validity of our results.

Utsunomiya et al. found a moderate correlation between MG and MVA after the MitraClip procedure in 97 patients with pre-existing pulmonary hypertension. Confirmatively, they discovered that intraprocedural MG predicts adverse outcomes in a cohort of comparable patients (20).

In a monocentric study including 51 elderly patients (mean age: 75 years), Boerlage-van Dijk et al. demonstrated that intraprocedural assessment of MG systematically underestimates the value compared to real life. 
Of note, the authors found no correlation between higher intraprocedural MG and increased heart failure symptoms at FU (9). In contrast, we found higher NYHA functional classes and lower six-minute walk distances at FU, as well as higher one-year mortality in patients with intraprocedural MG [?]4.5 $\mathrm{mmHg}$, which might be due to the fact that the majority of patients included in the cited study had FMR (74\%), and mostly suffer from chronic heart failure. This might hamper discerning persisting advanced heart failure symptoms and symptoms due to elevated MG at FU - high competing risk. Our cohort comprised a balanced number of MR etiologies (DMR: 40\%, FMR: 42.8\%, mixed: 17.2\%), which might be a reason for the divergent finding.

An intraprocedural MG $>4.4 \mathrm{mmHg}$ was shown by Patzelt et al. to be predictive for a combined endpoint consisting of all-cause mortality, redo procedure, and LVAD implantation after MitraClip only in patients with DMR, but not with FMR. Moreover, higher intraprocedural MG was correlated with lower functional capacity at FU in the same study. The authors found the patient's age to be the strongest independent predictor for the combined endpoint followed by residual MR $>$ II and intraprocedural MG (7). We also found that baseline MG and central clip implantation are relevant predictors for unfavourable intraprocedural MG, although we included more patients with FMR (42.5\%) and in more advanced stages of heart failure. We additionally found a negative influence of elevated intraprocedural MG on clinical outcomes regardless of MR aetiology.

Itabashi et al. showed that increased dimensions of the MV and the LV might be accountable for a somewhat lower intraprocedural MG in a comparable cohort after one-clip implantation (22). Contrary to our findings, patients with FMR showed a tendency for developing higher intraprocedural MG in this study, which might be due to smaller annular dimensions in their study cohort (AP diameter: $32 \mathrm{~mm}$ vs $38 \mathrm{~mm}$ ).

Of note, MR geometry has a direct influence on the development of elevated MG. We found LAI to be the strongest predictor for unfavourable MG after clip deployment. This new parameter, which reflects on the length of the leaflets in relation to annular dimensions and offers an adequate geometrical assessment of the MV, was not assessed in all of the studies cited but seemed to be associated with residual MR, MG, and outcomes after the MitraClip procedure (14).

Apart from residual MR as a well-known prognostic parameter, postinterventional MG appears to be an independent predictor for clinical outcomes despite in-hospital decrease to values below $4.5 \mathrm{mmHg}$. It shows a dynamic postinterventional process and is influenced by various haemodynamic parameters such as blood pressure, heart frequency, volume condition of the patient, sedation or anaesthesia, haemoglobin, inotropes. Therefore, its sporadic assessment may lead to under-or overestimations, which may lead to misinterpretation intrainterventionally. Understanding of MG dynamics and its predictors is desirable to get more favourable outcomes after a successful interventional MR reduction compared to just residual MR based decisionmaking. The definite pathomechanism of this clinical entity stays still unexplained as an encouraging reason for further prospective multicentric studies. Considering that higher postprocedural MG is associated with worse outcome, forthcoming procedural and device/system-related improvements are desirable.

\section{Limitations:}

This single-centre study has several limitations. It was performed with limited sample size and short FU duration. Echocardiographic analyses were not performed or validated by an independent core lab. Invasive assessment of the left atrial pressure or exercise echocardiography was not done. Further evaluation of the impact of MG on outcomes according to the aetiology of MR (FMR vs DMR) was not possible owing to the too low number of patients with elevated MG. To validate the predictive value of parameters for adverse outcomes and unfavourable MG appropriately powered multicentric studies with a larger patient cohort, a longer FU and subgroup and multivariate analysis according to cut-off values and/or propensity matching, are required.

\section{CONCLUSION:}

Intraprocedural MG was found to be a strong predictor for a high 12-month mortality rate (cut-off value: 
$4.5 \mathrm{mmHg}$ ) and adverse functional outcomes (cut-off value: $3.9 \mathrm{mmHg}$ ), irrespective of the aetiology of MR. In conformity, patients with an in-hospital persistent elevated $\mathrm{MG}$ ([?]4.5 $\mathrm{mmHg}$ ) showed the worst clinical results, including the lowest functional capacity and the highest 12-month mortality followed by patients with an in-hospital decrease in MG to values below $4.5 \mathrm{mmHg}$ at discharge. Additionally, MV geometry assessed by LAI ( $>1.11$ ) was the strongest predictor for unfavourable intraprocedural MG, followed by MG at baseline, the number of implanted clips and clip orientation (central implantation). These parameters might help for selecting appropriate patients, intrainterventional decision-making, and avoiding adverse MG values resulting in more favourable clinical outcomes.

Acknowledgement: We thank Dr Meghan Campbell (scientific coordinator in the Heart Center Bonn, University Hospital Bonn, Germany) for proofreading the manuscript.

Funding: This research received no grant from any funding agency in public, commercial or not-for-profit sectors.

Disclosures: Ozturk C, Sprenger Kim, Sugiura A, Weber M, Tabata N, Schueler R have no conflict of interest. Nickenig G has received speaker honoraria and research grants from Medtronic, Boston Scientific, Edwards Lifesciences, and Abbott.

\section{Author contributions:}

Concept/design: Can Ozturk, Robert Schueler

Data analysis/interpretation: Can Ozturk, Robert Schueler, Marcel Weber, Noriaki Tabata, Atsushi Sugiura

Drafting article: Can Ozturk, Robert Schueler, Georg Nickenig

Critical revision of the article: Robert Schueler, Georg Nickenig, Marcel Weber

Statistics: Can Ozturk, Kim Sprengler, Robert Schueler

Data collection: Kim Sprengler, Can Ozturk, Noriaki Tabata, Atsushi Sugiura

\section{References:}

1. Feldman T, Foster E, Glower DD, et al: Percutaneous repair or surgery for mitral regurgitation. N Engl J Med 2011;364(15):1395-406.

2. Franzen O, Baldus S, Rudolph V, et al: Acute outcomes of MitraClip therapy for mitral regurgitation in high-surgical-risk patients: emphasis on adverse valve morphology and severe left ventricular dysfunction. Eur Heart J 2010; 31(11):1373-81.

3. Guerin P, Bourguignon S, Jamet N, et al: MitraClip therapy in mitral regurgitation: a Markov model for the cost-effectiveness of a new therapeutic option. J Med Econ 2016;19(7):1-13.

4. Feldman T, Kar S, Elmariah S, et al: Randomized Comparison of Percutaneous Repair and Surgery for Mitral Regurgitation: 5-Year Results of EVEREST II. J Am Coll Cardiol 2015;66(25):2844-54.

5. Baumgartner H, Falk V, Bax JJ, et al: [2017 ESC/EACTS Guidelines for the management of valvular heart disease]. Kardiol Pol 2018;76(1):1-62.

6. Halaby R, Herrmann $\mathrm{H}$, Lim S, et al: LACK OF ASSOCIATION OF MITRAL VALVE GRADIENT AFTER MITRACLIP WITH OUTCOMES IN FUNCTIONAL MITRAL REGURGITATION: RESULTS FROM THE COAPT TRIAL. Journal of the American College of Cardiology , 75 (11 Supplement 1), 1309.

7. Patzelt J, Zhang W, Sauter R, et al: Elevated Mitral Valve Pressure Gradient Is Predictive of LongTerm Outcome After Percutaneous Edge-to-Edge Mitral Valve Repair in Patients With Degenerative Mitral Regurgitation ( MR ), But Not in Functional MR. Journal of the American Heart Association , 8 (13), e011366. 
8. Matsuzaki M, Tamitani M, Toma Y, et al: Mechanism of augmented left atrial pump function in myocardial infarction and essential hypertension evaluated by left atrial pressure-dimension relation. Am J Cardiol 1991;67(13):1121-1126.

9. Boerlage-van Dijk K, van Riel ACMJ, de Bruin-Bon RHACM, et al: Mitral inflow patterns after MitraClip implantation at rest and during exercise. J Am Soc Echocardiogr 2014;27(1):24-31.e1.

10. Huang FQ, Tan RS, Sim D, et al: Left ventricular diastolic function assessment using time differences between mitral annular velocities and transmitral inflow velocities in patients with heart failure. Heart Lung Circ 2015;24(3):257-63.

11. Seeger J, Muller P, Gonska B, et al: Percutaneous Mitral Valve Repair With the MitraClip in Primary Compared With Secondary Mitral Valve Regurgitation Using the Mitral Valve Academic Research Consortium Criteria. J Invasive Cardiol 2017;29(4):145-50.

12. Lancellotti P, Moura L, Pierard LA, et al: European Association of Echocardiography recommendations for the assessment of valvular regurgitation. Part 2: mitral and tricuspid regurgitation (native valve disease). Eur J Echocardiogr 2010:307-332.

13. Zoghbi WA, Enriquez-Sarano M, Foster E, et al: American Society of Echocardiography. Recommendations for evaluation of the severity of native valvular regurgitation with two-dimensional and Doppler echocardiography. J Am Soc Echocardiogr 2003:777-802.

14. Tabata N, Weber M, Sugiura A, et al: Impact of the Leaflet-to-Annulus Index on Residual Mitral Regurgitation in Patients Undergoing Edge-to-Edge Mitral Repair. JACC Cardiovasc Interv 2019;12(24):2462-2472.

15. Grasso C, Baldus S. The MitraClip transcatheter mitral valve repair system. EuroIntervention 2015;11 Suppl W(W): W45-6. Doi: 10.4244/EIJV11SWA11.

16. De Rosa R, Silverio A, Baldi C, et al: Transcatheter Repair of Functional Mitral Regurgitation in Heart Failure Patients - A Meta-Analysis of 23 Studies on MitraClip Implantation. Circ J 2018;82(11):2800-10.

17. Athappan G, Raza MQ, Kapadia SR. MitraClip Therapy for Mitral Regurgitation: Primary Mitral Regurgitation. Interv Cardiol Clin 2016;5(1):71-82.

18. Biaggi P, Felix C, Gruner C, et al: Assessment of mitral valve area during percutaneous mitral valve repair using the MitraClip system: comparison of different echocardiographic methods. Circ Cardiovasc Imaging 2013;6(6):1032-1040.

19. Neuss M, Schau T, Isotani A, et al: Elevated Mitral Valve Pressure Gradient After MitraClip Implantation Deteriorates Long-Term Outcome in Patients With Severe Mitral Regurgitation and Severe Heart Failure. JACC Cardiovasc Interv 2017;10(9):931-939.

20. Utsunomiya H, Itabashi Y, Kobayashi S, et al: Effect of Percutaneous Edge-to-Edge Repair on Mitral Valve Area and Its Association With Pulmonary Hypertension and Outcomes. Am J Cardiol 2017;120(4):662-9.

21. Thaden JJ, Malouf JF, Nkomo VT, et al: Mitral Valve Anatomic Predictors of Hemodynamic Success With Transcatheter Mitral Valve Repair. J Am Heart Assoc 2018;7(2):1117.

22. Itabashi Y, Utsunomiya H, Kubo S, et al: Different indicators for postprocedural mitral stenosis caused by single- or multiple-clip implantation after percutaneous mitral valve repair. J Cardiol 2018;71(4):336-345.

\section{Figure Legends}

Figure 1: Flow chart for dynamic changes of MG: peri-interventionally and during FU

Figure 2: Kaplan-Meier curve for 12-month mortality in MG groups 
Figure 3: Graphs of the probability of functional outcomes (A) and 12-month mortality (B) in group 1, group $2 \mathrm{~A}$, and group $2 \mathrm{~B}$

Figure 4: Negative relationship between unfavourable intraprocedural MG and the leaflet-to-annulus index (LAI). The results from a multivariate analysis, which compared the predictors of unfavourable intraprocedural MG were depicted as a table.

\section{Supplementary data}

Supplemental Figure 1: Bar graph of intraprocedural MG according to clip orientation with an illustration of the orientation of clip implantations. The results of variance analysis for the assessment of the impact of clip localisation on intraprocedural MG in patients with one-clip approach were presented as a table.

Supplemental Figure 2: Bar graph of intraprocedural MG according to the aetiology of MR

\section{Hosted file}

Tables.pdf available at https://authorea.com/users/393304/articles/506958-dynamics-of-themean-transmitral-pressure-gradient-and-its-impact-on-clinical-outcomes-after-mitraclip

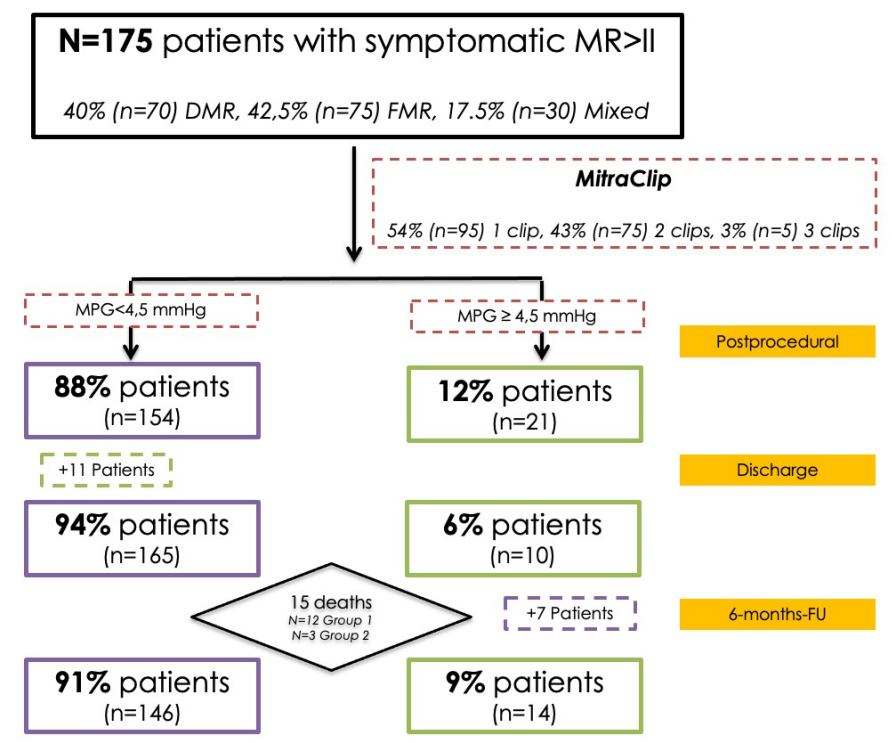



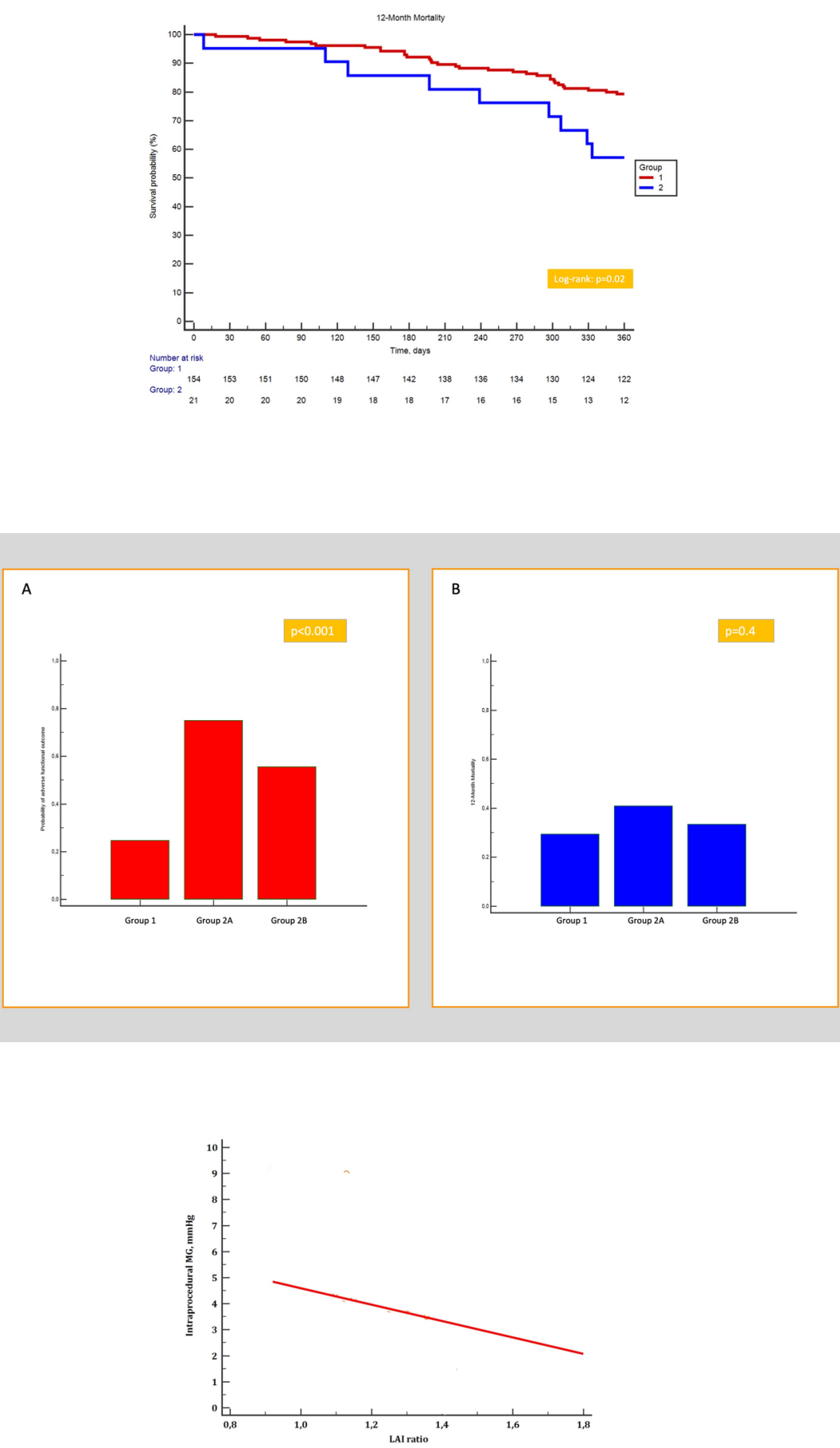

\begin{tabular}{|c|c|c|c|}
\hline Parameters & OR & $95 \%$ CI & p-Value \\
\hline LAI & 9.74 & 0.43 to 217.17 & 0.001 \\
\hline Implanted dips $>2$ & 7.54 & 1.24 to 87.67 & 0.01 \\
\hline MPG at baseline & 2.06 & 1.58 to 2.68 & 0.02 \\
\hline Central clip implantation & 1.87 & 0.67 to 3.45 & 0.05 \\
\hline
\end{tabular}

